\title{
DETERMINAÇÃO DE PONTOS OTIMOS PARA LOCALIZAÇÃO E IMPLANTAÇÃO DE USINAS DE BIODIESEL NO ESTADO DO TOCANTINS
}

\section{DETERMINATION FOR GREAT FOR LOCATION AND IMPLEMENTATION OF BIODIESEL IN TOCATINS STATE}

\author{
Karyn Siebert Pinedo* E-mail: karynpinedo@yahoo.com.br \\ Yolanda Vieira de Abreu* E-mail: yolandaabreu@gmail.com.br \\ *Universidade Federal do Tocantins, UFT, Palmas, TO
}

Resumo: O objetivo do artigo foi de mostrar, uma análise, feita no Estado do Tocantins a fim de determinar pontos ótimos para implantação de usinas de biodiesel. Para essa analise foram consideradas varáveis como transporte, estoque, armazenamento, manuseio de materiais entre outros, para que se conseguir um resultado satisfatório para novos empreendimentos. Sabe-se que hoje para se conseguir um bom resultado as empresas têm que focar uma analise rigorosa na cadeia da logística, que têm como objetivo tornar disponíveis produtos e serviços no local onde são necessários, no momento em que são desejados ao menor custo possível. Para a obtenção dos resultados aqui apresentados utilizou-se do modelo matemático - Programação Linear Inteira Mista (PLIM) - e a posteriori técnica de cenários.

Palavras-chave: Biodiesel. Logística. Otimização. Programação Linear Inteira Mista (PLIM). cenários.

\begin{abstract}
The objective was to show an analysis made in the state of Tocantins to determine optimal points for the implementation of biodiesel plants. For this analysis were considered variables such as transportation, inventory, warehousing, material handling among others, to achieve a satisfactory outcome for new ventures. We know that today to get a good result, businesses have to focus on a rigorous analysis of the logistics chain, which are designed to make available products and services in the location where needed, when they are wanted at the lowest possible cost. To obtain the results presented here we used the mathematical model - Mixed Integer Linear Programming (MILP) - technique and retrospective scenarios.
\end{abstract}

Key-words: Biodiesel. Logistics. Optimization. Mixed Integer Linear Programming (MILP). Scenarios.

\section{INTRODUÇÃO}

Estamos em uma nova era de mudança e inovações tecnológicas, empresariais e de paradigmas sociais e ambientais. As mudanças de gestão empresarial trazem idéias que são capazes de gerar grandes impactos tanto na área interna da empresa, quanto na externa (ambiente). Pode se observar uma preocupação cada vez maior com o meio ambiente e o bem-estar do 
trabalhador e sua adequação ao ambiente de trabalho. As novas tecnologias ligadas à informática contribuem para que o trabalhador possa desempenhar cada vez mais suas funções podendo escolher realizá-las dentro da empresa ou em sua casa.

As empresas e indústrias de diversos setores de atividades utilizam-se da logística dentro do processo estratégico para manter a eficiência das operações de produção e distribuição. A logística empresarial integra de forma coordenada todos esses fatores, incluindo a instalação de fábricas, distribuidoras e estudo de viabilidade econômica (logística) de pontos ótimos de localização. O transporte de cargas é um serviço fundamental, na cadeia de produção e distribuição de bens industriais e agrícolas, e se destaca na economia brasileira porque o país tem dimensões continentais. A preocupação das empresas e indústrias em relação à localização está muito relacionada com a disponibilidade de matéria-prima, insumos e alcance da distribuição para os pontos de consumo de seu produto.

Para a realização do estudo sobre a localização ótima de usinas de biodiesel escolheu-se como estudo de caso o Estado do Tocantins. Utilizaramse dados primários e secundários dos municípios como, por exemplo: custo de transporte, demografia, produção de oleaginosas, dados estatísticos sobre a pecuária e dados dos modais de transporte. O resultado deste trabalho tem como objetivo apresentar os municípios que poderiam receber as novas usinas de biodiesel, com os menores custos de transporte da matéria-prima e próximo de centros consumidores do produto final.

Para se alcançar o objetivo deste artigo foi necessário minimizar uma função objetivo dada pela Programação Linear Inteira Mista (PLIM). A função objetivo foi determinada pelas variáveis de produção, localização, comercialização, custos com transporte e coeficientes de ponderação dos dados. Inicialmente foram consideradas, de modo geral, três variáveis primárias: (i) Zonas de consumo; (ii) Municípios produtores de matéria-prima; e (iii) Localização de modais de transporte. Foram escolhidos os municípios que de algum modo estavam dentro dos três princípios gerais para escolha dos pontos ótimos de localização. 


\section{PROBLEMAS DE LOCALIZAÇÃO}

A análise dos problemas de localização é uma área em estudo desde o início do século passado. O primeiro modelo de localização foi proposto por Alfred Weber em 1909, e dominou por muitos anos a literatura. No entanto, a área unificada de estudo chamada "Localização de Instalações" emerge na década de 1960; as pesquisas de Hakimi, publicadas em 1964, estabelecem resultados importantes na teoria da localização e gerou grande interesse entre os pesquisadores (HAKIMI, 1964).

Os problemas de localização, na sua forma mais geral, podem ser descritos como segue. Um conjunto de clientes espacialmente distribuídos em uma área geográfica requer certo produto ou serviço específico. A demanda dos clientes deve ser coberta por uma ou mais instalações. As instalações podem operar dentro de um quadro de cooperação ou de competição, dependendo dos produtos ou serviços requeridos pelos clientes. O processo de decisão estabelece onde devem localizar suas instalações no território desejado, tendo em conta as exigências do cliente e as restrições geográficas.

Dentre os problemas de localização podem ser identificados três elementos essenciais. As instalações, que denotam um conjunto de objetos a serem localizados para prestar um serviço ou produto. Os locais, que se referem a um conjunto de todos os pontos possíveis para colocar instalações. Finalmente, os clientes que são os usuários finais e necessitam determinados serviços ou produtos (OWEN, 1994).

O termo localização é usado para denotar uma variedade de bens e serviços e também pontos de instalação ou localização ótima de edifícios como: armazéns, fábricas, escolas, hospitais, shoppings, edifícios públicos, com a finalidade de otimizar sua relação com outros já existentes. As principais propriedades que caracterizam estas instalações são: o número e tipo. Em diversos modelos de localização, o número de instalações é fixado a priori. No caso em que se determina localizar uma só uma instalação, em um determinado espaço ou território, é um problema de instalação simples. Porém, 
no caso geral, o modelo considera, simultaneamente, várias instalações e é chamado de problema de multi-instalação (OWEN, 1994).

Outra propriedade importante para as instalações é dada pelo seu tipo, está especifica suas características tais como: a capacidade de serviço e considerações sobre sua estrutura. Nos casos simples, as questões de localização requerem instalações idênticas para prestar o mesmo serviço ou produto. Os modelos de localização, também, podem ser diferenciados de acordo com o serviço simples e multi-serviços, com base na capacidade da instalação para fornecer um ou mais tipos de serviços ou produtos. Alguns problemas de localização admitem instalações onde são considerados que a capacidade é ilimitada, enquanto outros buscam a melhor localização, com uma produção limitada. Portanto, as questões de localização também podem ser classificadas como capacitadas ou não capacitadas (OWEN, 1994).

Ainda segundo o autor o elemento essencial para os modelos de localização é o local físico onde se irá localizar a instalação. O conjunto de pontos ótimos de localização é comumente chamado o espaço de solução e pode-se representar de modo contínuo, discreto ou na forma de rede os quais são:

- Espaço Discreto: Quando se pode especificar uma lista de lugares possíveis para localizar instalações. Nesse caso, se oferece flexibilidade, pois é possível incorporar ao modelo características do tipo geográficas e econômicas.

- Espaço Contínuo: São problemas considerados no espaço euclidiano. O caso mais típico, considerado um espaço euclidiano bidimensional.

- Representação de redes: Para muitas aplicações, nas quais se consideram serviços públicos e privados, são estudados problemas de localização em que se deve operar com uma infra-estrutura de certa rede (rede rodoviária, rede fluvial, rede ferroviária, rede de aeroportos, oleodutos. etc), geralmente representado por um gráfico. Os problemas de rede podem ser discretos ou contínuos, comumente as estações de serviço podem estar localizadas nas extremidades ou vértices do gráfico que representa a contrapartida Revista Produção Online. Florianópolis, SC, v.11, n. 4, p. 1160-1181, out./dez. 2011. 
da infra-estrutura de rede. Quando um modelo é definido por uma representação de rede, o gráfico pode assumir diferentes formas: (i) Grafo direcionado (ii) Grafo não-direcionado.

Para Rezende (2003), os problemas de localização surgem da necessidade de localizar centros para a satisfação ótima da procura de um conjunto de clientes. A palavra "cliente" é usada para designar objetos que requerem acesso a um serviço ou demanda do produto. $\mathrm{Na}$ tentativa de analisar problemas de localização, deve interagir com os clientes, por isso é necessário conhecer a sua distribuição, aplicação e compartilhamento.

- Para distribuição deve-se assumir que a carga, por cliente, está uniformemente distribuída ou localizada em um ponto específico ou nos vértices de uma rede.

- No caso da demanda, a cada cliente é atribuído um valor que expressa a quantidade de serviço exigido. A ação pode representar a quantidade de produto ou serviço solicitado por um usuário ou uma área ou região geográfica. Em ambos os casos, não podem ser conhecidos com certeza.

- A última característica do cliente é o seu comportamento. Em alguns casos, o cliente é livre para escolher com qual instalação deseja ser servido, e conduz à pergunta: Será que o cliente sempre vai para a instalação mais próxima ou utiliza de outros critérios e preferências para escolher a instalação? Além disso, os clientes podem agir individualmente ou em grupos.

Um problema clássico de localização é o problema da p-mediana. Esse problema, na sua forma mais simples é caracterizado pelo tipo de instalações e sua localização com o cliente. As instalações a localização não têm restrições de capacidade. O número de instalações é definido de acordo com um parâmetro $p$, e oferecem o mesmo tipo de serviço. Os clientes exigem uma quantia fixa de bens ou serviços, conforto e sempre escolhem para ser atendidos, pela instalação mais próxima de sua localização. A relação com os locais é expressa através de uma função distância que representa o caminho mais curto na rede para chegar sua localização (REZENDE, 2003). 
Com alguma freqüência, empresários ou grandes investidores se deparam com situações onde existe a necessidade de escolher alternativas favoráveis. Algumas vezes, estas escolhas podem se restringir ao conhecimento de determinados conceitos de custos. No entanto, existem outras situações onde os empresários ou grandes investidores enfrentam problemas relacionados à limitação dos recursos de produção, de distribuição, das vendas, e de outros fatores (ROSA, 2005).

Nesses casos, os empresários necessitam de ferramentas mais apropriadas, de modo a contornar esses fatores limitativos para tomar decisões, controlar as operações e simular desempenhos. Neste caso se faz necessário à utilização da Programação Linear como uma técnica matemática, que permitirá o subsídio na escolha da melhor decisão.

Em qualquer ramo do saber, uma teoria é um conjunto de idéias, experiências, propriedades, fenômenos causais, teoremas, etc. de modo que torne o todo coerente. Estas teorias são desenvolvidas com o intuito de explicar um maior número de fatos ou fenômenos mediante leis gerais ou universais. Toda teoria, pela sua natureza, é abrangente e geral.

\begin{abstract}
Partindo da realidade observada, analisada através de uma ótica coerente com uma determinada postura teórica, procura-se identificar os elementos e relações relevantes do sistema. Com esses elementos e relações estruturadas através de linguagens formais matemática, analógica, gráfica etc. Constroem-se uma representação do sistema real, que é chamada de modelo (ALVARENGA, 2001).
\end{abstract}

Segundo Alvarenga (2001), um modelo apresenta apenas uma visão ou cenário de um fragmento do todo. Normalmente, para estudar um determinado fenômeno complexo, criam-se vários modelos com a intenção de construir um quadro simplificado da realidade. Um modelo matemático é uma representação ou interpretação simplificada da realidade, ou uma interpretação de um fragmento de um sistema, segundo uma estrutura de conceitos mentais ou experimentais. Os modelos matemáticos são utilizados praticamente em todas as áreas científicas, como, por exemplo, na biologia, química, física, economia, engenharia e na própria matemática pura.

$\mathrm{Na}$ aplicação de modelos é comum usar de algoritmos e processos heurísticos. Um algoritmo é uma seqüência finita de instruções bem definidas e não-ambíguas; cada uma das quais pode ser executada mecanicamente num 
período de tempo finito e com uma quantidade de esforço finita. O conceito de algoritmo é freqüentemente ilustrado quando, por exemplo, utilizamos de uma receita de cozinha, embora muitos algoritmos sejam mais complexos. Eles podem repetir passos, fazer interações ou necessitar de decisões (tais como comparações ou lógica) até que a tarefa seja completada (SCHWARTZ, 1999).

Para que um algoritmo seja corretamente desenvolvido e executado tem que estar com suas premissas adequadas ao problema real a ser solucionado. Um algoritmo não representa, necessariamente, um programa de computador, e sim os passos necessários para realizar uma tarefa.

\footnotetext{
É essencial que estas premissas sejam adequadas ao sistema. Caso contrário, as soluções encontradas poderão ser matematicamente ótimas, porém não representam a realidade das operações (BANDEIRA, 2006).
}

A Pesquisa Operacional (PO) ou Investigação Operacional (IO) é um ramo interdisciplinar da matemática aplicada que faz uso de modelos matemáticos, estatísticos e de algoritmos na ajuda à tomada de decisões e problemas de logística. É usada, sobretudo, para analisar sistemas complexos do mundo real, tipicamente com o objetivo de melhorar ou aperfeiçoar o desempenho.

Neste artigo, optou-se por usar um modelo matemático - PLIM - para se alcançar os objetivos propostos, que é de determinar pontos ótimos de localização e usinas de biodiesel no Estado do Tocantins.

\section{MÉTODO DE PESQUISA UTILIZADO}

Para a realização deste artigo utilizou-se da Programação Linear Inteira Mista (PLIM), operacionalizada no Excel, isto permitiu determinar os pontos ótimos para implantação da usina de biodiesel. Para a escolha dos dados a serem utilizados no modelo matemático e realização dos cenários, utilizaramse como premissas: 1) Municípios com mais de 10.000 mil habitantes ou que possuam classificação de atividade econômica como média ou alta renda, segundo o IBGE (2009); 2) Cada município comporta somente uma usina; 3) Municípios produtores de oleaginosas e sebo para matéria-prima; 4) Descentralização da produção; 5) Municípios próximos de modais de 
transporte; 6) Distância máxima de $500 \mathrm{~km}$ da usina até a cidade considerada como zona de consumo; 7) Capacidade de produção, da usina, máxima 10.000 mil litros por semana; 8) Todas as usinas com mesmo custo de instalação; 9) Mesma capacidade de produção e estoque para todas as usinas; 10) Quando o fornecedor de matéria prima, a zona de consumo e a usina coincidirem no mesmo ponto (município) considerou-se $1 \mathrm{~km}$ para o deslocamento entre elas.

A Programação Linear Inteira Mista (PLIM) é a metodologia mais comum usada em modelos de localizações comerciais, a grande vantagem da programação inteira é permitir que sejam incluídos na análise os custos fixos, bem como diferentes níveis de custos variáveis para as instalações. A variável continua corresponde às variáveis de processo (temperatura, peso, medida, distância, etc.). Os modelos baseados na programação linear inteira mista têm solução mais complexa em relação àqueles que utilizam à programação linear (HAFFNER 2007).

Com os dados de população e classificação econômica dos principais pontos de distribuição (municípios do Tocantins) obtivemos um grupo de 32 pontos. Para esses pontos de distribuição foi estimado que a quantidade de veículos para consumo de biodiesel, seria multiplicada por 0,125 vezes o número de habitantes. Por exemplo, no município de Araguaína para um total de 115.759 habitantes, teríamos 14.702 veículos que seriam os consumidores do biodiesel. Os 32 pontos de zona de consumo serão classificados em nossa função objetivo com o indicador $i=1,2,3, \ldots, 30,31,32$.

Segundo IBGE (2007) observa-se que o município de Araguaína tem a maior concentração de grãos e frigoríficos. Tendo este município grande potencial para instalação de usina, fornecer a matéria prima também e ser um grande consumidor de seu produto final. Após serem considerados os municípios pela sua capacidade de produção de matéria-prima, os mesmos foram, também, analisados pela sua localização geográfica, considerando o transporte terrestre, fluvial e ferroviário, assim como também os locais próximos à produção de matéria prima, consideramos os 26 pontos ótimos como se mostra na tabela 3.1. Cada fornecedor da matéria prima identifica-se com um código numérico $k=1,2,3, \ldots, 23,24,25,26$ : 
Esses municípios além de terem capacidade de produção de matériaprima, sua localização propicia o escoamento do produto final e o recebimento de matéria-prima, utilizando um ou vários tipos de modais disponíveis no Estado, assim alguns estão próximos da rodovia, outros da hidrovia e assim por diante. Na tabela 3.1 mostra alguns pontos que podem fornecer matéria prima.

Tabela 3.1 - Pontos ótimos em condições de fornecer matéria prima para a fabricação o biodiesel.

\begin{tabular}{clcl}
\hline $\begin{array}{c}\text { Variável } \\
\text { k= }\end{array}$ & $\begin{array}{c}\text { Fornecedor de matéria } \\
\text { prima }\end{array}$ & $\begin{array}{c}\text { Variável } \\
\mathrm{k}=\end{array}$ & $\begin{array}{l}\text { Fornecedor de matéria } \\
\text { prima }\end{array}$ \\
\hline 1 & Araguaina & 14 & Formoso do Araguaia \\
2 & Nova Olinda & 15 & Araguaçu. \\
3 & Palmeirante & 16 & Lagoa da confusão \\
4 & Darcinópolis. & 17 & Dianápolis \\
5 & Gurupi & 18 & Santa Rosa do Tocantins \\
6 & Brejinho de Nazaré & 19 & Mateiros \\
7 & Figueirópolis & 20 & Campos lindos \\
8 & Alvorada & 21 & Porto Nacional \\
9 & Guarai & 22 & Monte do Carmo \\
10 & Brasilândia do Tocantins & 23 & Pedro Afonso \\
11 & Tupirama & 24 & Bom Jesus do Tocantins \\
12 & Tupiratins & 25 & Silvanópolis \\
13 & Presidente Kennedy & 26 & Santa Maria do Tocantins \\
\hline
\end{tabular}

Fonte: IBGE (2009)

O ponto ótimo para instalação de uma usina de biodiesel será aquele que possa cumprir os requisitos de proporcionar o menor custo de instalação, maior facilidade com menor custo de escoamento da produção, assim como o recebimento da matéria-prima e estar perto do centro de consumo e da aquisição de mão-de-obra.

Pela análise o modelo adequado para nosso propósito é dado pela função objetivo

$$
Z=\sum_{j=1}^{25} \sum_{k=1}^{26} C_{k j} U_{k j}+\sum_{j=1}^{25} \sum_{i=1}^{32} D_{i j} W_{i} X_{j i}+\sum_{j=1}^{25} \sum_{r=1}^{R} F_{i j} Y_{r j}
$$

Nosso objetivo é minimizar nossa "função objetivo", isto é, estudar o resultado da função:

mín.Z $=\sum_{j=1}^{25} \sum_{k=1}^{26} C_{k j} U_{k j}+\sum_{j=1}^{25} \sum_{i=1}^{32} D_{i j} w_{i} X_{j i}+\sum_{j=1}^{25} \sum_{r=1}^{R} F_{i j} Y_{r j}$ 
As restrições para este modelo são:

$\sum_{\mathrm{j}=1}^{25} \mathrm{X}_{\mathrm{ij}}=1 \quad \forall \mathrm{i}=1,2,3, \ldots, 32$

Esta equação (3.3) restringe o abastecimento da demanda de cada zona de consumo i por algumas usinas j (fornecedores), aqui consideramos a produção total semanal como um todo, representado pela unidade. O município de Araguaína fica somente com o $80 \%$ de sua produção e distribui os outros 20\% para o município de Wanderlândia.

A previsão da demanda de Palmas é de 60 mil litros por semana fornecida pelas usinas de: Peixe 8.000 litros, Lagoa da confusão 10.000 litros; Dianápolis 1.000 litros Arraias 3.000 litros; Natividade 8.000 litros; Conceição do Tocantins 10.000 litros; Santa Rosa do Tocantins 10.000 litros;São Felix do Tocantins 10.000 litros

$$
\sum_{j=1}^{25} w_{i} X_{i j} \leq \sum_{r=1}^{R} r_{j} Y_{i j} \quad \forall j=1,2,3, \ldots, 25
$$

A restrição representada pela equação (3.4) garante que a demanda das zonas de consumo seja inferior à capacidade entregue pelos fornecedores $\mathrm{j}$. Estamos trabalhando com uma previsão de entrega diferenciada para diferentes municípios.

$$
\sum_{r=1}^{R} r_{j} Y_{r j} \leq 1 \quad \forall j=1,2,3, \ldots, 25
$$

A equação (3.5) permite que, no máximo 10.000 litros de capacidade semanal sejam atribuídos a cada usina. Em todo o trabalho estamos considerando a capacidade de produção $r_{j}$ constante para todas as usinas, pois todas têm a mesmas características de produção e tem estoque suficiente para a entrega.

$$
\sum_{\mathrm{i}=1}^{32} \mathrm{w}_{\mathrm{i}} \mathrm{X}_{\mathrm{ij}} \leq \sum_{\mathrm{k}=1}^{26} \mathrm{U}_{\mathrm{kj}} \quad \forall \mathrm{j}=1,2,3, \ldots, 25
$$

A equação (3.6) assegura que a soma da demanda do das zonas de consumo i não ultrapasse a capacidade de produção da usina j com a matéria prima recebida de todos os fornecedores $\mathrm{k}$, supor que com cinco toneladas de matéria prima se obtenha 10.000 litros de biodiesel... 
$\sum_{\mathrm{j}=1}^{25} \mathrm{U}_{\mathrm{kj}} \leq \mathrm{vk} \quad \forall \mathrm{k}=1,2,3, \ldots, 26$

A equação (3.7) garante que a quantidade do produto distribuída pela usina j seja inferior à capacidade de produção do fornecedor de matéria prima $\mathrm{k}$.

$Y_{\mathrm{rj}}, \in\{0,1\}$

A equação (3.8) é a variável binária inteira positiva, será um, se a usina j com capacidade $r$ tem biodiesel para pronta entrega; zero caso contrário.

$$
\mathrm{U}_{\mathrm{ij}} \geq 0 \quad \forall \mathrm{i}=1,2,3, \ldots, 22, \quad \forall \mathrm{j}=1,2,3, \ldots, 25
$$

A equação (3.9) indica que a quantidade de produto transportado do fornecedor k para a usina j (toneladas), sempre existirá. Será considerada uma distância mínima de um quilômetro quando o fornecedor k da matéria prima é do mesmo ponto da instalação da usina j.

Sabe-se que a variável $C_{k j}$ representa o custo unitário de transporte por tonelada e quilômetro transportado de matéria prima do fornecedor $\mathrm{k}$ para a usina j, ao preço de mercado $\mathrm{R} \$ 0,75$ tonelada a cada quilômetro transportado.

Vamos supor que quantidade transportada de matéria prima $U_{k j}$ constante igual a cinco toneladas diárias ( $\mathrm{k}$ para a usina j), assim obtemos $\mathrm{C}_{\mathrm{kj}} \mathrm{U}_{\mathrm{kj}}$ que será a primeira componente de nossa função objetivo (3.1). O custo parcial ocasionado pelo transporte do fornecedor $k$, ate a usina j mostra-se na tabela 3.2 .

Sendo a variável $U_{\mathrm{kj}}=5$ toneladas a quantidade de produto transportado do fornecedor k para a usina j (em toneladas) teremos o custo preliminar. Um resultado parcial mostra-se a seguir. 
Tabela 3.2 - Custo total de transporte da matéria prima do fornecedor $\mathbf{k}$ para a usina $\mathbf{j}$ (R\$/5000quilos)

Fornecedor de matéria prima

\begin{tabular}{|c|c|c|c|c|c|c|c|c|c|}
\hline & $\mathbf{C}_{\mathbf{j k}} \mathbf{U}_{\mathbf{j k}}$ & $\begin{array}{l}\frac{\pi}{5} \\
\frac{.}{\pi} \\
\frac{\pi}{2} \\
\frac{\pi}{2}\end{array}$ & 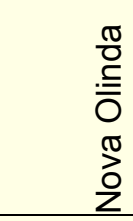 & 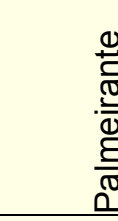 & 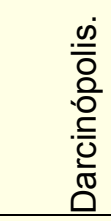 & $\begin{array}{l}\overline{2} \\
\frac{2}{2} \\
\overline{0} \\
0\end{array}$ & 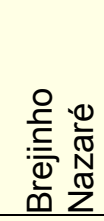 & 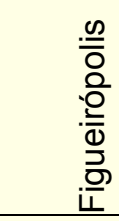 & $\begin{array}{l}\frac{\pi}{0} \\
\frac{\pi}{0} \\
\frac{0}{2}\end{array}$ \\
\hline \multirow{11}{*}{ 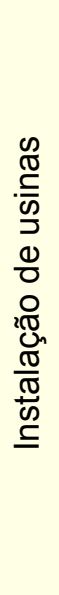 } & Araguaina & 3,75 & 217,5 & 360 & 322,5 & 2005 & 1755 & 2560 & 2340 \\
\hline & Arapoema & 550 & 375 & 520 & 725 & 1870 & 1555 & 2060 & 2220 \\
\hline & Tocantinópolis & 625 & 760 & 730 & 255 & 2550 & 2215 & 2770 & 2885 \\
\hline & Araguatins & 950 & 1165 & 1045 & 505 & 2955 & 2730 & 3000 & 3335 \\
\hline & Gurupi & 2005 & 1790 & 1855 & 2635 & 3,75 & 397,5 & 206,5 & 337,5 \\
\hline & Alvorada & 2340 & 2125 & 2200 & 2710 & 337,5 & 715 & 176,5 & 3,75 \\
\hline & Peixe & 2280 & 2065 & 1940 & 2730 & 274 & 461,5 & 319 & 367,5 \\
\hline & Guarai & 725 & 505 & 510 & 1060 & 1285 & 1025 & 1545 & 1695 \\
\hline & Araguacema & 1695 & 1480 & 890 & 1300 & 1250 & 1140 & 1540 & 1700 \\
\hline & Caseara & 1975 & 1860 & 1170 & 1550 & 1065 & 1045 & 1400 & 1565 \\
\hline & Palmeirópolis & 2970 & 2755 & 2650 & 2905 & 740 & 970 & 535 & 412,5 \\
\hline
\end{tabular}

A tabela 3.3 mostra um primeiro resultado parcial para a equação (3.1), isto representa custo em reais $\mathrm{R} \$$ do transporte da matéria prima do fornecedor $\mathrm{k}$ para a usina $\mathrm{j}$, todos os fornecedores entregam a mesma quantidade em toneladas a todas as 25 usinas. Para o caso de um fornecedor $\mathrm{k}$ da matéria prima tiver que entregar à usina j situada no mesmo local, foi considerado uma distância fixa de um quilômetro. 
Tabela 3.3 - Custo total de transporte da matéria prima do fornecedor k para cada ponto da usina j.

\begin{tabular}{|c|c|c|}
\hline$\sum_{k=1}^{26} C_{k j} U_{k j}$ & Instalação de usinas & $\begin{array}{l}\text { Custo do transporte da } \\
\text { matéria prima em } R \$ \text { reais }\end{array}$ \\
\hline \multirow{5}{*}{ 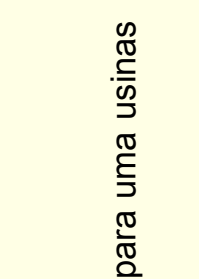 } & Araguaina & $35.993,75$ \\
\hline & Arapoema & $34.254,00$ \\
\hline & Tocantinópolis & $48.410,00$ \\
\hline & Araguatins & $59.175,00$ \\
\hline & Gurupi & $29.719,25$ \\
\hline \multirow{5}{*}{ 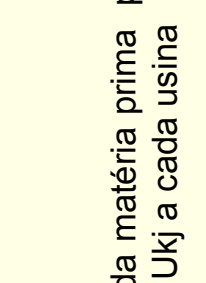 } & Alvorada & $35.434,25$ \\
\hline & Peixe & $31.764,50$ \\
\hline & Guarai & $23.829,75$ \\
\hline & Araguacema & $31.655,00$ \\
\hline & Caseara & $32.950,00$ \\
\hline \multirow{2}{*}{$\begin{array}{l}x \\
\frac{0}{0} \\
\frac{\pi}{0} \\
\frac{\pi}{0}\end{array}$} & Palmeirópolis & $42.272,50$ \\
\hline & Tupiratins & $25.285,75$ \\
\hline \multirow{2}{*}{ 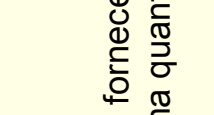 } & Lagoa da confusão & $31.002,75$ \\
\hline & Formoso do Araguaia & $32.095,75$ \\
\hline \multirow{11}{*}{ 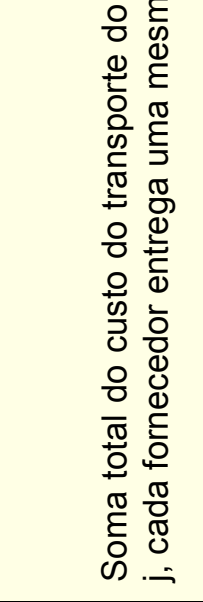 } & Araguaçu. & $43.663,75$ \\
\hline & Dianápolis & $36.703,75$ \\
\hline & Arraias & $49.855,00$ \\
\hline & Paraná & $38.575,00$ \\
\hline & Natividade & $31.069,50$ \\
\hline & Conceição do Tocantins & $37.500,00$ \\
\hline & Santa Rosa do Tocantins & $29.113,25$ \\
\hline & São Felix do Tocantins & $31.241,50$ \\
\hline & Campos lindos & $44.823,75$ \\
\hline & Porto Nacional & $27.850,75$ \\
\hline & Santa Maria do Tocantins & $28.715,75$ \\
\hline
\end{tabular}

Na seguinte etapa calculam-se os custos com transporte do biodiesel para as zonas de consumo.

Consideremos a variável $0<\mathrm{X}_{\mathrm{ij}} \leq 1$, se a zona de consumo i recebe 0 produto da usina j (máxima distância entre elas de $500 \mathrm{~km}$ ); $\mathrm{X}_{\mathrm{ij}}=0$, caso contrário (mínima distância entre elas $501 \mathrm{~km}$ ) ou para o casso que a zona de consumo i não receba produto da usina j. Na tabela 3.4 foi realizada uma analise de quanto cada município precisará para ficar abastecido do biodiesel. 
Tabela 3.4 - Zona de consumo.

Zona de consumo

Consideremos $\mathrm{Xji}=1$, se a zona de consumo $\mathrm{i}$ recebe o produto da usina $\mathrm{j}$ (máxima distância entre elas de $500 \mathrm{~km}$ ); zero, caso contrário

\begin{tabular}{|c|c|c|c|c|c|c|c|c|c|c|c|c|c|c|c|}
\hline & $\mathbf{X}_{\mathrm{ij}}$ & 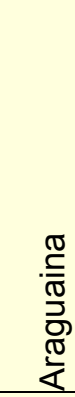 & $\begin{array}{l}\frac{\pi}{0} \\
. \overline{0} \\
0 \\
\frac{\pi}{2} \\
0\end{array}$ & 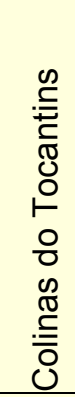 & 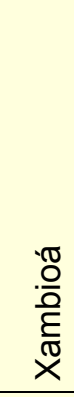 & 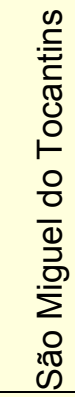 & 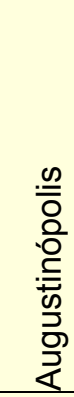 & 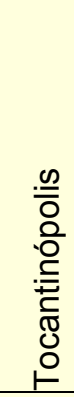 & $\begin{array}{l}\stackrel{0}{C} \\
\stackrel{\bar{\sigma}}{\pi} \\
\frac{\sigma}{\sigma} \\
\frac{\pi}{\alpha}\end{array}$ & $\begin{array}{l}\bar{\alpha} \\
\frac{2}{2} \\
\overline{0}\end{array}$ & $\begin{array}{l}\bar{\pi} \\
\bar{\pi} \\
\widetilde{J} \\
0\end{array}$ & 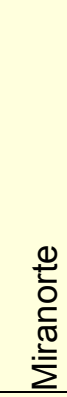 & 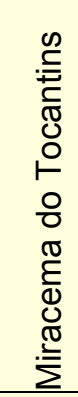 & $\begin{array}{l}\frac{\pi}{\pi} \\
\frac{\pi}{\sigma} \\
\frac{\pi}{\pi} \\
\frac{\pi}{\alpha} \\
0 \\
0 \\
0 \\
0 \\
0 \\
0 \\
0 \\
4\end{array}$ & 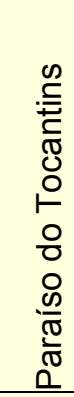 \\
\hline \multirow{13}{*}{ 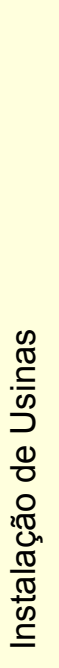 } & Araguaina & 0,8 & 0,0 & 0,0 & 0,0 & 0,0 & 0,0 & 0,0 & 0,0 & 0,0 & 0,0 & 0,0 & 0,0 & 0,0 & 0,0 \\
\hline & Arapoema; & 0,0 & 0,0 & 0,5 & 0,0 & 0,0 & 0,3 & 0,0 & 0,0 & 0,0 & 0,0 & 0,0 & 0,0 & 0,0 & 0,0 \\
\hline & Tocantinópolis & 0,1 & 0,0 & 0,0 & 0,0 & 0,0 & 0,0 & 0,5 & 0,4 & 0,0 & 0,0 & 0,0 & 0,0 & 0,0 & 0,0 \\
\hline & Araguatins & 0,0 & 0,0 & 0,0 & 0,0 & 0,3 & 0,0 & 0,0 & 0,5 & 0,0 & 0,0 & 0,0 & 0,0 & 0,0 & 0,0 \\
\hline & Gurupi & 0,1 & 0,0 & 0,3 & 0,0 & 0,0 & 0,0 & 0,0 & 0,0 & 0,6 & 0,0 & 0,0 & 0,0 & 0,0 & 0,0 \\
\hline & Alvorada & 0,0 & 0,0 & 0,0 & 0,0 & 0,0 & 0,0 & 0,0 & 0,0 & 0,7 & 0,0 & 0,0 & 0,0 & 0,0 & 0,0 \\
\hline & Peixe & 0,0 & 0,0 & 0,0 & 0,0 & 0,0 & 0,0 & 0,0 & 0,0 & 0,2 & 0,0 & 0,0 & 0,0 & 0,0 & 0,0 \\
\hline & Guarai & 0,0 & 0,2 & 0,0 & 0,0 & 0,0 & 0,0 & 0,0 & 0,0 & 0,0 & 0,6 & 0,0 & 0,0 & 0,0 & 0,0 \\
\hline & Araguacema & 0,8 & 0,0 & 0,0 & 0,0 & 0,0 & 0,0 & 0,0 & 0,0 & 0,0 & 0,0 & 0,2 & 0,0 & 0,0 & 0,0 \\
\hline & Caseara & 0,0 & 0,0 & 0,0 & 0,2 & 0,0 & 0,0 & 0,0 & 0,0 & 0,0 & 0,1 & 0,0 & 0,6 & 0,0 & 0,0 \\
\hline & Palmeirópolis & 0,0 & 0,0 & 0,0 & 0,0 & 0,0 & 0,0 & 0,0 & 0,0 & 0,0 & 0,0 & 0,0 & 0,0 & 0,0 & 0,5 \\
\hline & Tupiratins & 0,2 & 0,1 & 0,2 & 0,0 & 0,0 & 0,0 & 0,0 & 0,0 & 0,0 & 0,0 & 0,2 & 0,0 & 0,0 & 0,0 \\
\hline & Lagoa da confusão & 0,0 & 0,0 & 0,0 & 0,0 & 0,0 & 0,0 & 0,0 & 0,0 & 0,0 & 0,0 & 0,0 & 0,0 & 0,0 & 0,0 \\
\hline
\end{tabular}

Fonte: Elaboração dos autores

Por exemplo, para uma usina instalada em Araguaína do total de sua produção 80\% (8.000 litros) fica no ponto de consumo de Araguaína, os outros $20 \%$ (2.000 litros) são distribuídos para Wanderlândia, assim temos $X_{11}=0,8$ e $\mathrm{X}_{1(25)}=0.2$, como a usina localizada em Araguaína não distribui para os outros pontos de consumo tem-se que $X_{i j}=0$. A usina de Tupiratins distribui para Araguaína $20 \%$, logo $X_{(12) 1}=0,2$, para Nova Olinda $10 \%$, logo $X_{(12) 2}=0.1$, para Colinas do Tocantins 20\% logo $X_{(12)(11)}=0.2$, para Miranorte $20 \%$ logo $\mathrm{X}_{(12)(11)}=0.2$, também distribui para Araguanã e Wanderlândia.

Os dados da $\mathrm{w}_{\mathrm{j}}$ da tabela 3.4 são calculados tendo como referencial a população de cada município , mediante o fator de $20 \%$ do total da população 
para as zonas de consumo que representam os principais municípios com um bom desenvolvimento econômico e o fator de 13,33\% para as outras zonas de consumo. Este número de veículos se multiplica pela quantidade média de consumo de biodiesel semanal e obtemos a tabela 3.5 que mostra a quantidade de biodiesel necessário para abastecer cada um dos pontos da zona de consumo.

Tabela 3.5 - Quantidade wi de biodiesel em litros semanais necessário para cada zona de consumo

\begin{tabular}{clcclc}
\hline $\mathrm{i}=$ & $\begin{array}{l}\text { Município/ } \\
\text { Zona de consumo }\end{array}$ & wi & i & $\begin{array}{l}\text { Município/ } \\
\text { Zona de consumo }\end{array}$ & wi \\
\hline 1 & Araguaina & 23.152 & 17 & Paraná & 2.098 \\
2 & Nova Olinda & 2.104 & 18 & Taguatinga & 2.822 \\
3 & Colinas do Tocantins & 5.860 & 19 & Goiatins. $\left(^{*}\right)$ & 1.552 \\
4 & Xambioá $\left({ }^{*}\right)$ & 1.447 & 20 & Porto Nacional & 9.058 \\
5 & São Miguel do Tocantins & 2.044 & 21 & Pedro Afonso & 2.059 \\
6 & Augustinópolis $\left({ }^{*}\right)$ & 1.973 & 22 & Palmas & 35.677 \\
7 & Tocantinópolis $\left({ }^{*}\right)$ & 2.845 & 23 & Talismã & 511 \\
8 & Araguatins & 5.195 & 24 & Alvorada & 1.599 \\
9 & Gurupi & 14.283 & 25 & Wanderlândia & 1.863 \\
10 & Guarai & 4.334 & 26 & Palmeirópolis & 1.624 \\
11 & Miranorte & 27 & Araguanã & 1.000 \\
12 & Miracema do Tocantins & 3.937 & 28 & Arapoema $\left({ }^{*}\right)$ & 1.310 \\
13 & Formoso do Araguaia $\left({ }^{*}\right)$ & 2.430 & 29 & Natividade $\left({ }^{*}\right)$ & 1.212 \\
14 & Paraíso do Tocantins & 8.058 & 30 & Ananás $\left({ }^{*}\right)$ & 1.248 \\
15 & Dianápolis $\left({ }^{*}\right)$ & 2.478 & 31 & Araguaçu(*) & 1.199 \\
16 & Arraias $\left({ }^{*}\right)$ & 32 & Sítio Novo do Tocantins $\left({ }^{*}\right)$ & 1.242 \\
\hline
\end{tabular}

O preço a pagar por litro de combustível transportado é de $\mathrm{R} \$ 0,0002$ $\mathrm{km} /$ litro transportado, a variável $\mathrm{C}_{\mathrm{kj}}$ indica estes custos entre os pontos diversos.

O produto do custo unitário $D_{\mathrm{ij}}$ de transporte da usina j para a zona de consumo i (R\$/metro cúbico), pela demanda wi por produto na zona de consumo i (unid/período) vezes o fator $X_{i j}$ representa o custo total de 
transporte de cada usina $\mathrm{j}$ ao ponto de distribuição i, este produto denotado por $D_{i j} w_{i} X_{i j}$ e indica os custos de distribuição de uma usina a todos os 32 pontos de zona de consumo. A soma de todos estes custos representada por $\sum_{i=1}^{32} D_{i j} w_{i} X_{j i}$

É o custo total de transporte de cada usina a todas as zonas de consumo, o resultado desta soma pode-se apreciar na tabela 3.6.

Tabela 3.6 - Parciais dos custos de transporte do biodiesel das zonas de produção as zonas de consumo

\begin{tabular}{|c|c|c|}
\hline$\sum_{i=1}^{32} D_{i j} w_{i} X_{j i}$ & Instalação de usinas & $\begin{array}{ll}\text { Custo } & \text { do } \\
\text { transporte } & \text { da } \\
\text { matéria prima } & \text { em } \\
\mathrm{R} \$ \text { reais } & \end{array}$ \\
\hline \multirow{25}{*}{ 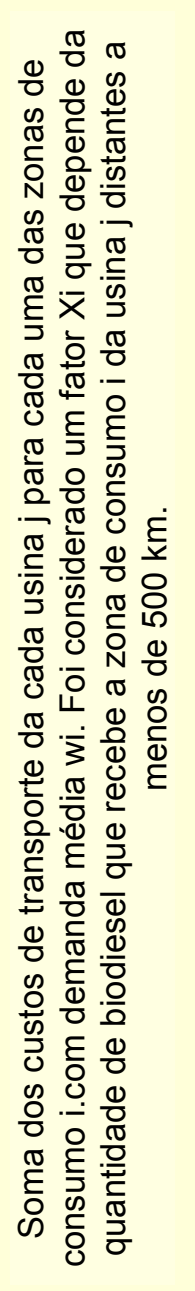 } & Araguaina & $22.471,16$ \\
\hline & Arapoema; & $115.512,92$ \\
\hline & Tocantinópolis & $143.536,34$ \\
\hline & Araguatins & $15.130,30$ \\
\hline & Gurupi & $407.132,60$ \\
\hline & Alvorada & $180.663,74$ \\
\hline & Peixe & $186.2660,44$ \\
\hline & Guarai & $29.636,88$ \\
\hline & Araguacema & $169.3613,28$ \\
\hline & Caseara & $161.640,40$ \\
\hline & Palmeirópolis & $146.452,66$ \\
\hline & Tupiratins & $201.363,90$ \\
\hline & Lagoa da confusão & $777.758,60$ \\
\hline & Formoso do Araguaia & $110.665,44$ \\
\hline & Araguaçu. & $446.672,00$ \\
\hline & Dianápolis & $330.242,22$ \\
\hline & Arraias & $937.138,10$ \\
\hline & Paraná & $383.000,08$ \\
\hline & Natividade & $133.5989,28$ \\
\hline & Conceição do Tocantins & $204.0724,40$ \\
\hline & Santa Rosa do Tocantins & $107.7445,40$ \\
\hline & São Felix do Tocantins & $152.6975,60$ \\
\hline & Campos lindos & $120.3904,00$ \\
\hline & Porto Nacional & $9.058,00$ \\
\hline & Santa Maria do Tocantins & $142.6163,20$ \\
\hline
\end{tabular}


Com os dados da tabela 3.5 e tabela 3.6 obtêm a tabela 3.7 que indica os custos para obter a matéria prima e distribuir o biodiesel pronto para 0 consumo.

Tabela 3.7 - Valores mínimos para a função objetivo.

\begin{tabular}{|c|c|c|c|}
\hline Instalação de usinas & $\sum_{k=1}^{26} C_{k j} U_{k j}$ & $\sum_{i=1}^{32} D_{i j} w_{i} X_{j i}$ & $=\mathrm{Z}$ \\
\hline Araguaina & $359.93,75$ & $224.71,16$ & $584.64,91$ \\
\hline Arapoema & $34.254,00$ & $115.512,92$ & $149.766,90$ \\
\hline Tocantinópolis & $48.410,00$ & $143.536,34$ & $191.946,30$ \\
\hline Araguatins & $59.175,00$ & $15.130,30$ & $74.305,30$ \\
\hline Gurupi & $29.719,25$ & $407.132,60$ & $436.851,90$ \\
\hline Alvorada & $35.434,25$ & $180.663,74$ & $216.098,00$ \\
\hline Peixe & $31.764,50$ & $1.862 .660,44$ & $189.4425,00$ \\
\hline Guarai & 23829,75 & $29.636,88$ & $53.466,63$ \\
\hline Araguacema & 31655,00 & $169.3613,28$ & $1.725 .268,00$ \\
\hline Caseara & 32950,00 & 161640,40 & $194.590,40$ \\
\hline Palmeirópolis & 42272,50 & 146452,66 & 188725,20 \\
\hline Tupiratins & 25285,75 & 201363,90 & 226649,70 \\
\hline Lagoa da confusão & 31002,75 & 777758,60 & 808761,40 \\
\hline Formoso do Araguaia & 32095,75 & 110665,44 & 142761,20 \\
\hline Araguaçu. & 43663,75 & 446672,00 & 490335,80 \\
\hline Dianápolis & $36.703,75$ & 330242,22 & 366946,00 \\
\hline Arraias & $49.855,00$ & $937.138,10$ & $986.993,10$ \\
\hline Paraná & $38.575,00$ & $38.300,08$ & 421575,10 \\
\hline Natividade & $31.069,50$ & $1.335 .989,28$ & 1367059,00 \\
\hline Conceição do Tocantins & $37.500,00$ & $2.040 .724,40$ & $2.078 .224,00$ \\
\hline Santa Rosa do Tocantins & $29.113,25$ & $1.077 .445,40$ & $1.106 .559,00$ \\
\hline São Felix do Tocantins & $31.241,50$ & $1.526 .975,60$ & $1.558 .217,00$ \\
\hline Campos lindos & $44.823,75$ & $1.203 .904,00$ & $1.248 .728,00$ \\
\hline Porto Nacional & $27.850,75$ & $9.058,00$ & $36.908,75$ \\
\hline Santa Maria do Tocantins & $28.715,75$ & $142.6163,20$ & $1.454 .879,00$ \\
\hline
\end{tabular}

Fonte: Elaboração dos autores a partir da aplicação do modelo.

Esses resultados da tabela acima se obtiveram a partir do primeiro e do segundo resultado parcial. A estes custos parciais devem-se adicionar os custos de instalação e preços da cada uma das usinas a serem instaladas.

Considerando que todas as usinas j têm as mesmas características da sua fabricação, implantação e capacidade de produção $r$, logo todas elas têm um custo fixo de $F_{r j}$ constante. Também suporemos que todas as usinas j têm Revista Produção Online. Florianópolis, SC, v.11, n. 4, p. 1160-1181, out./dez. 2011. 
capacidade $r=10.000$ litros de biodiesel para pronta entrega semanalmente, neste caso a variável binária $Y_{r j}=1$, logo se têm o terceiro resultado esperado

$$
\sum_{r=1}^{R} F_{r j} Y_{r j}
$$

igual a uma constante $\mathrm{C}$ para todo usina $\mathrm{j}=1,2,3, \ldots, 24,25$. Assim, obtemos a partir da tabela 3.8:

Tabela 3.8 - Valor total de instalação de cada usina, obtidos da tabela 3.7.

$$
\text { Instalação de usinas } \quad Z=\sum_{k=1}^{26} C_{k j} U_{k j}+\sum_{i=1}^{32} D_{i j} w_{i} X_{i j}+\sum_{j=1}^{25} \sum_{r=1}^{R} F_{r j} Y_{r j}
$$

Araguaina

Arapoema

Tocantinópolis

Araguatins

Gurupi

Alvorada

Peixe

Guarai

Araguacema

Caseara

Palmeirópolis

Tupiratins

Lagoa da confusão

Formoso do Araguaia

Araguaçu.

Dianápolis

Arraias

Paraná

Natividade

Conceição do Tocantins

Santa Rosa do Tocantins

São Felix do Tocantins

Campos lindos

Porto Nacional

Santa Maria do Tocantins

$$
\begin{aligned}
& 584.64,91+C \\
& 149.766,90+C \\
& 191.946,30+C \\
& 74.305,30+C \\
& 436.851,90+C \\
& 216.098,00+C \\
& 189.4425,00+C \\
& 53.466,63+C \\
& 1.725 .268,00+C \\
& 194.590,40+C \\
& 188725,20+C \\
& 226649,70+C \\
& 808761,40+C \\
& 142761,20+C \\
& 490335,80+C \\
& 366946,00+C \\
& 986.993,10+C \\
& 421575,10+C \\
& 1367059,00+C \\
& 2.078 .224,00+C \\
& 1.106 .559,00+C \\
& 1.558 .217,00+C \\
& 1.248 .728,00+C \\
& 36.908,75+C \\
& 1.454 .879,00+C \\
& 10
\end{aligned}
$$

Fonte: Elaboração dos autores a partir da aplicação do modelo.

Os valores dos custos de instalação de cada usina mostram-se na tabela 3.9. Os custos da instalação esta determinado pela variável $Z$ (custo total de Revista Produção Online. Florianópolis, SC, v.11, n. 4, p. 1160-1181, out./dez. 2011. 
implantação) mais $\mathrm{C}$, que representa os custos de instalação (prédio, máquinas, equipamentos e outros que são necessários para deixar as instalações prontas para começar a ser utilizada) de cada uma das usinas.

Tabela 3.9 - Relação de municípios (em ordem crescente de valores) para implantação de usinas no Estado.

\begin{tabular}{|c|c|}
\hline Instalação de usinas & $\min Z=$ \\
\hline Porto Nacional & $=36.908,75+C$ \\
\hline Araguaina & $=58.464,91+C$ \\
\hline Guaraí & $=53.466,63+C$ \\
\hline Araguatins & $=74.305,30+C$ \\
\hline Formoso do Araguaia & $=142.761,20+C$ \\
\hline Arapoema & $=149.766,90+C$ \\
\hline Palmeirópolis & $=188.725,20+C$ \\
\hline Tocantinópolis & $=191 \cdot 946,30+C$ \\
\hline Caseara & $=194.590,40+C$ \\
\hline Alvorada & $=216.098,00+C$ \\
\hline Tupiratins & $=226.649,70+C$ \\
\hline Dianápolis & $=366.946,00+C$ \\
\hline Paraná & $=421.575,10+C$ \\
\hline Gurupi & $=436.851,90+C$ \\
\hline Araguaçu. & $=490.335,80+C$ \\
\hline Lagoa da confusão & $=808.761,40+C$ \\
\hline Arraias & $=986.993,10+C$ \\
\hline Santa Rosa do Tocantins & $=1106559,00+C$ \\
\hline Campos lindos & $=1248728,00+C$ \\
\hline Natividade & $=1367059,00+C$ \\
\hline Santa Maria do Tocantins & $=1454879,00+C$ \\
\hline São Felix do Tocantins & $=1558217,00+C$ \\
\hline Araguacema & $=1725268,00+C$ \\
\hline Peixe & $=1894425,00+C$ \\
\hline Conceição do Tocantins & $=2078224,00+C$ \\
\hline
\end{tabular}

Fonte: Elaboração dos autores a partir da aplicação do modelo.

A tabela 3.9 está indicando os valores de vinte e cinco municípios para a implantação de usinas. Desses vinte e cinco municípios foi determinado que apenas os dez primeiros com seus respectivos custos seriam foco de nossa análise.

Revista Produção Online. Florianópolis, SC, v.11, n. 4, p. 1160-1181, out./dez. 2011. 


\section{ANÁLISE DOS RESULTADOS OBTIDOS}

Dos 25 pontos (municípios) escolhidos para análise no modelo matemático, somente os dez foram considerados como pontos ótimos para a implantação das usinas. Tal determinação levou em consideração que estes propiciam um custo menor de instalação. No mapa 4.1, pode-se observar a localização dos que foram considerados, para este cenário.

\section{Mapa 4.1}

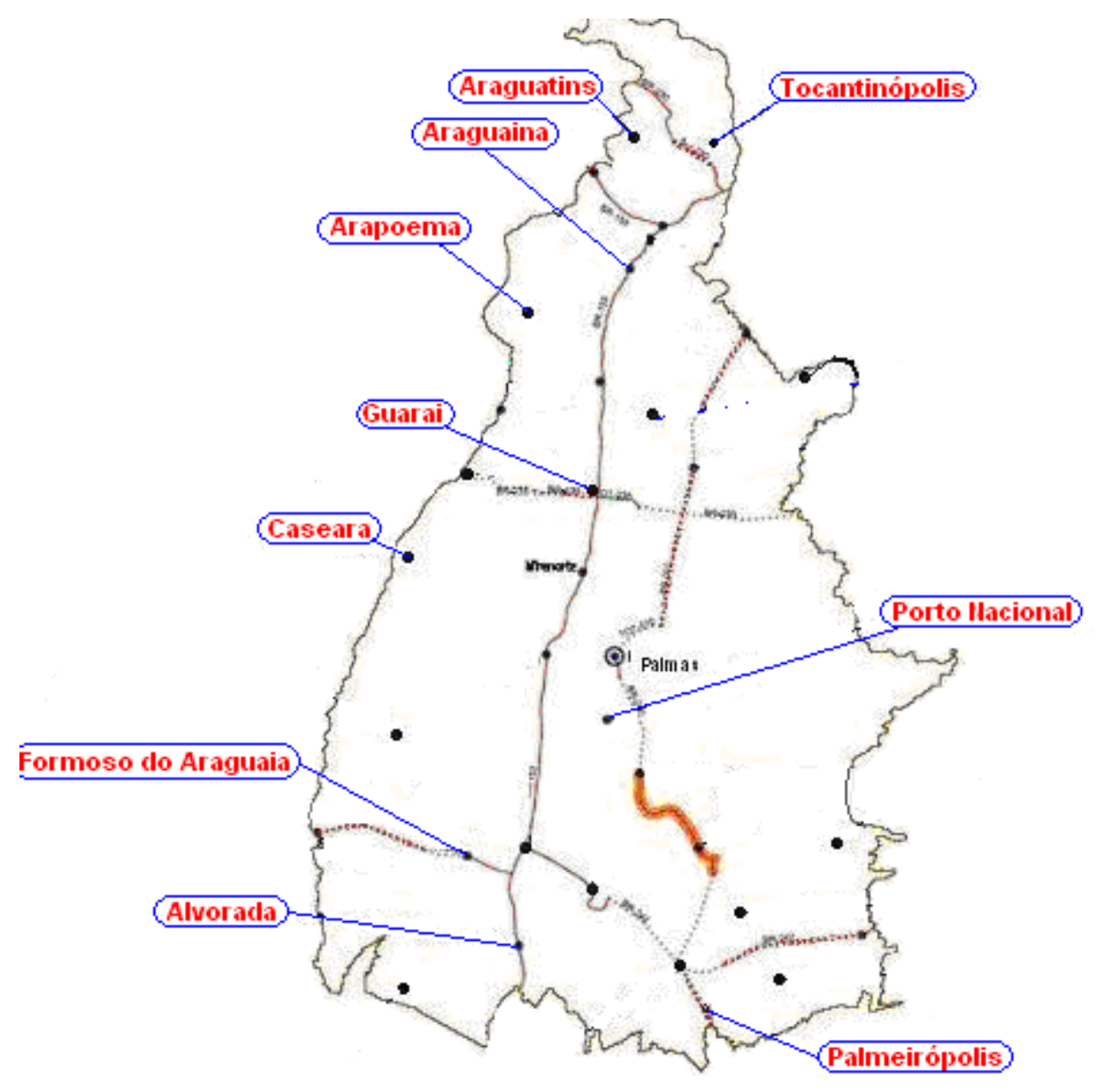

Esses pontos representam um menor custo de instalação para dez primeiras usinas como mostra na tabela 3.9. Esses pontos de localização de usinas poderão distribuir para os seguintes municípios com o menor custo possível. 


\section{CONCLUSÃO}

Para a realização deste artigo utilizou-se do um modelo matemático Programação Linear Inteira Mista, operacionalizada no Excel para determinar os pontos ótimos para implantação da usina de biodiesel. Para determinação e execução do modelo utilizaram-se como premissas para implantação da usina de biodiesel: 1) os municípios (pontos ótimos) com acima de 10.000 mil habitantes; 2) foram eliminados os municípios que ficaram com demografia muito acima ou abaixo da media e também os que já possuíam usinas não poderiam ser considerados para implantação das novas usinas; 3) municípios produtoras de matéria-prima para produção de biodiesel; 4) descentralização da produção de biodiesel; 5) municípios pertos de modais de transporte; 6) foi considerada a entrega de 5 toneladas de matéria prima semanal pelo fornecedor de matéria prima; 7) até a distancia de $500 \mathrm{~km}$ da usina ate a cidade considerada como zona de consumo; 8) a usina implantada produz ate 10.000 mil litros por semana de biodiesel, 9) Um mesmo preço de custo de construção da usina em relação a prédios e outros itens para cada usina, 11) a mesma capacidade de produção e estoque para cada usina, 12) Quando o fornecedor da matéria prima, a zona de consumo e a usina no mesmo ponto foi considerada $1 \mathrm{~km}$ para o deslocamento entre elas..

Este estudo baseou-se no referencial teórico sobre logística e teoria da localização para justificá-lo. Os municípios tocantinenses escolhidos para a execução do modelo matemático foram inicialmente 33 , o modelo matemático depois de aplicado utilizando os dados pré-estabelecidos pela pesquisadora e por sua orientadora, apresentou os 25 municípios com maior potencial para o recebimento das novas usinas de biodiesel. Esses municípios foram, Porto Nacional, (2) Araguaina, (3) Guarai, (4) Araguatins, (5) Formoso do Araguaia, (6) Arapoema, (7) Palmeirópolis, (8) Tocantinópolis, (9) Caseara, (10) Alvorada, (11) Tupiratins, (12) Dianápolis, (13) Paraná, (14) Conceição do Tocantins, (15)Gurupi,(16) Araguaçu., (17) Lagoa da confusão, (18) Arraias, (19) Santa Rosa do Tocantins, (20) Campos lindos, (21)Natividade, (22) Santa Maria do Tocantins, (23) São Felix do Tocantins , (24)Araguacema,, (25) Peixe, 
Outros estudos relacionados à logística, modelo PLIM e estudo de localização de usinas de biodiesel poderão ser elaborados no futuro. $O$ presente estudo delimitou-se a considerar somente a matéria-prima oleaginosa e sebo.Porém, os resultados obtidos foram satisfatórios e corresponderam as expectativas dos pesquisadores envolvidos.

\section{REFERENCIAS}

ALVARENGA, A.C. Logística aplicada, suprimento e distribuição física. São Paulo: Pioneira, 2001.

BANDEIRA Renata A. de M. Proposta de um sistema de Análise para Localização de Depósitos. Dissertação de Mestrado. Universidade Federal do Rio Grande do Sul. Escola de Engenharia. Programa de Pós-Graduação em Engenharia da Produção, 2006. 150p.

CAIXETA-FILHO José V. Pesquisa operacional: técnicas aplicadas a sistema agroindustriais. 2. ed. São Paulo: Atlas 2004, p. 170.

HAKIMI, S. L. Optimum location of switching centers and the absolute centers and medians of a graph. Operations Research, v.12, p.450-459, 1964. .

OWEN, Mal. SPC and continuous improvement. 1 ed. USA: IFS Publications, 1994.

RESENDE, E. Situação atual dos transportes no Brasil. Revista Transportes Agora, 2003. Disponível em: <http://www.transportes.gov.br/ascom/transporte Agora/>. Acesso em: 05 jun. 2009.

ROSA, Denise P., 2005. O Planejamento de centros logísticos com base na agregação de valor por serviços logísticos em terminais de transportes. (Tese de Doutorado).

SCHWARTZ, J. Air pollution and daily maortality in Birminghan, Alabama, American Journal of Epidemiology, Baltimore, v. 137, n.10, p.1136-1147, 1999.

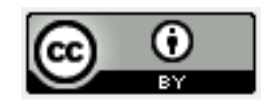

Artigo recebido em 16/10/2010 e aceito para publicação em 26/09/2011. 